\title{
Article \\ A Growth Differentiation Factor 15-Based Risk Score Model to Predict Mortality in Hemodialysis Patients
}

\author{
Jia-Feng Chang ${ }^{1,2,3,4,5}$, Po-Cheng Chen ${ }^{6}$, Chih-Yu Hsieh ${ }^{1,7}$ ) and Jian-Chiun Liou ${ }^{7, *}$ \\ 1 Division of Nephrology, Department of Internal Medicine, En Chu Kong Hospital, \\ New Taipei City 237, Taiwan; cjf6699@gmail.com (J.-F.C.); fish37435@hotmail.com (C.-Y.H.) \\ 2 Department of Nursing, Yuanpei University of Medical Technology, Hsinchu 300, Taiwan \\ 3 Division of Nephrology, Department of Internal Medicine, Shuang Ho Hospital, Taipei Medical University, \\ New Taipei City 235, Taiwan \\ 4 Graduate Institute of Aerospace and Undersea Medicine, Department of Medicine, \\ National Defense Medical Center, Taipei 114, Taiwan \\ 5 Renal Care Joint Foundation, New Taipei City 220, Taiwan \\ 6 Department of Urology, En Chu Kong Hospital, New Taipei City 237, Taiwan; b90401049@ntu.edu.tw \\ 7 School of Biomedical Engineering, Taipei Medical University, Taipei 110, Taiwan \\ * Correspondence: jcliou@tmu.edu.tw
}

check for updates

Citation: Chang, J.-F.; Chen, P.-C.; Hsieh, C.-Y.; Liou, J.-C. A Growth Differentiation Factor 15-Based Risk Score Model to Predict Mortality in Hemodialysis Patients. Diagnostics 2021, 11, 286. https://doi.org/ 10.3390/diagnostics11020286

Academic Editor: Lorenz Risch

Received: 10 December 2020

Accepted: 7 February 2021

Published: 11 February 2021

Publisher's Note: MDPI stays neutral with regard to jurisdictional claims in published maps and institutional affiliations.

Copyright: (c) 2021 by the authors. Licensee MDPI, Basel, Switzerland. This article is an open access article distributed under the terms and conditions of the Creative Commons Attribution (CC BY) license (https:// creativecommons.org/licenses/by/ $4.0 /)$.

\begin{abstract}
Background: The risk of cardiovascular (CV) and fatal events remains extremely high in patients with maintenance hemodialysis (MHD), and the growth differentiation factor 15 (GDF15) has emerged as a valid risk stratification biomarker. We aimed to develop a GDF15-based risk score as a death prediction model for MHD patients. Methods: Age, biomarker levels, and clinical parameters were evaluated at study entry. One hundred and seventy patients with complete information were finally included for data analysis. We performed the Cox regression analysis of various prognostic factors for mortality. Then, age, GDF15, and robust clinical predictors were included as a risk score model to assess the predictive accuracy for all-cause and $\mathrm{CV}$ death in the receiver operating characteristic (ROC) curve analysis. Results: Age, GDF15, and albumin were significantly associated with higher all-cause and CV mortality risk that were combined as a risk score model. The highest tertile of GDF-15 (>1707.1 pg/mL) was associated with all-cause mortality (adjusted hazard ratios (aHRs): 3.06 (95\% confidence interval (CI): 1.20-7.82), $p<0.05$ ) and CV mortality (aHRs: 3.11 (95\% CI: $1.02-9.50), p<0.05)$. The ROC analysis of GDF-15 tertiles for all-cause and CV mortality showed 0.68 (95\% CI $=0.59$ to 0.77 ) and $0.68(95 \% \mathrm{CI}=0.58$ to 0.79$)$, respectively. By contrast, the GDF15-based prediction model for all-cause and CV mortality showed 0.75 (95\% CI: 0.67-0.82) and 0.72 (95\% CI: 0.63-0.81), respectively. Conclusion: Age, GDF15, and hypoalbuminemia predict all-cause and CV death in MHD patients, yet a combination scoring system provides more robust predictive powers. An elevated GDF15-based risk score warns clinicians to determine an appropriate intervention in advance. In light of this, the GDF15-based death prediction model could be developed in the artificial intelligence-based precision medicine.
\end{abstract}

Keywords: growth differentiation factor 15; death prediction model; hemodialysis

\section{Introduction}

Patients receiving maintenance hemodialysis (MHD) have an exceedingly high risk of fatal events, and cardiovascular diseases (CVD) still top list of death causes [1]. Persistent, low-grade inflammation accompanied by malnutrition acts as a hallmark feature of chronic kidney disease (CKD), being involved in the pathogenesis of CVD and the development of all-cause mortality of MHD patients [2,3]. Growth differentiation factor-15 (GDF15), also known as macrophage inhibitory cytokine-1 belonging to the transforming growth factor $\beta$ superfamily, is a stress-induced protein [4]. In general population, circulating levels of GDF15 are highly elevated in response to pathological conditions such as oxidative stress, 
inflammation, tissue injury, metabolic dysregulation, autoimmune diseases, cancer entities, and hypoxia-related cardiovascular (CV) ailments [5]. In CKD patients, GDF15 levels are notably raised and responsible for the greater risks of CKD progression, CV morbidity, and mortality [6-10].

Whilst much has been done to better understand risk factors for fatal events in CKD patients, novel biomarkers and conventional risk stratification tools do not adequately predict prognosis and prevent mortality. Accordingly, fatality remains alarmingly high in CKD despite global age-standardized mortality being decreased by $30.4 \%$ for CVD, $14.9 \%$ for cancer and $41.3 \%$ for chronic obstructive pulmonary disease [11]. Such uncertainty of CVD progression and sudden death aggravates patients and clinicians as well. In light of this, a recent study integrates age, GDF15 biomarker, and clinical parameters as the ABC death risk score for clinical evaluation of patient safety and decision tree [12]. There has been increasing interest in identifying the novel death risk score model to effectively predict poor prognosis in CKD patients [13-15]. Emerging evidence has shown that the prognostic questionnaires of mortality in CKD and specifically in MHD patients serve as useful risk prediction tools for the early detection of clinical events. Currently, a series of practical questionnaires exist, including SARC-F (sluggishness, assistance in walking, rising from a chair, climb stairs, falls), KDQOL (Kidney Disease Quality of Life) instrument, Karnofsky Performance Status Scale, and 36-Item Short Form Survey (SF-36) [16]. In addition to the above prognostic questionnaires of mortality, a novel biomarker-based risk score system could be developed as an alternative screening tool. With regard to huge prevalence of CV morbidity and mortality in MHD populations, we aimed to assess the predictive validity of GDF15-based risk scores to develop a reliable early warning system for timely management of clinical events. Therefore, various risk factors for all-cause and CVD-related mortality in MHD patients were estimated using a Cox regression model and receiver operating characteristic (ROC) analysis in the present study.

\section{Materials and Methods}

\subsection{Participants in the Cohort}

The study had been approved by the Research Ethics Review Committee of En Chu Kong Hospital (ECKIRB1070102) in accordance with the ethical standards of the committee and the Helsinki declaration for research in humans. The written informed consent was obtained from the participants of this study. The relevant details of the research methods were described previously [1,3]. Patients undergoing MHD treatment for at least three months were eligible for inclusion. All patients had to be older than 18 years of age and be receiving thrice-weekly MHD, and 188 patients in end-stage kidney disease were included. Eighteen patients were excluded from the study because of inadequate dialysis, terminal illness, active infections, advanced cancer, active hepatitis, severe protein-energy wasting, incomplete data, or unwilling to participate.

\subsection{Assessment of Exposures}

The plasma GDF15 and bio-clinical parameters were ascertained at baseline. Plasma concentrations of GDF15 were measured by a commercial quantitative enzyme-linked immunosorbent (ELISA) assay (Human GDF15 ELISA Kit (Catalog Number: DGD15), R\&D Systems, Minneapolis, MN, USA) in accordance with the manufacturers' instructions. Plasma GDF15 concentrations were categorized into tertiles: low, medium, and high levels for tertiles 1, 2, and 3, respectively. In the primary analyses, we examined the association between GDF15 levels, relevant clinical parameters, all-cause, and CV mortality in the Cox regression model. The predictive value of GDF15 tertiles for all-cause and CV mortality was examined using the ROC analysis. In the secondary analyses, we used a simple scale system to determine GDF15-based death risk score: GDF15 tertiles 1 as score 1, tertiles 2 as score 2 , and tertiles 3 as score 3 . To strengthen the predictive value of GDF15-based score system, the significant clinical predictors in primary analyses were combined with GDF15 score as a GDF15-based death prediction model. The score (1 or 0 ) of the clinical 
predictors was defined by the cut-off value in the ROC analysis. All patients were then stratified by the GDF15-based risk score system into new categories. The predictive value of GDF15-based risk score system for all-cause and CV mortality was examined using the ROC analysis.

\subsection{Assessment of Covariates}

The following bio-demographic and laboratory parameters of each patient were recorded at baseline: age, gender, hypertension, diabetes mellitus (DM), previous CVD, hemodialysis (HD) vintage, systolic blood pressure, diastolic blood pressure, pre-dialysis blood urea nitrogen, normalized protein catabolic rate, creatinine, potassium, calcium, phosphorus, alkaline phosphatase (ALP), alanine aminotransferase, albumin, uric acid, total cholesterol, triglyceride, hemoglobin, platelet, and intact parathyroid hormone (iPTH). Previous CV diseases (CVD) were defined as diseases attributable to myocardial ischemia and infarction (ICD-10-CM Diagnosis Code: I20-I25), heart failure (ICD-10-CM Diagnosis Code: I50.1-I50.9), symptomatic or life-threatening arrhythmia (ICD-10-CM Diagnosis Code: I49), cerebrovascular diseases (ICD-10-CM Diagnosis Code: I60-I69), pulmonary embolism (ICD-10-CM Diagnosis Code: I26), and peripheral artery diseases (ICD-10-CM Diagnosis Code: I73). The HD vintage was defined as the duration of time between the first day of HD treatment and the first day that the patient entered the cohort. The blood pressure was recorded in the horizontal recumbent position before a midweek dialysis session. Pre-dialysis blood samples were obtained from the existing vascular access for the further analyses. We adjusted plasma calcium according to the following equation: adjusted calcium, measured calcium $+((4.0$-plasma albumin in $\mathrm{g} / \mathrm{dL}) \times 0.8)$. All laboratory tests were performed by the standard procedures with certified methods.

\subsection{Ascertainment of Outcomes}

CV mortality in study patients was defined as death attributable to myocardial ischemia and infarction, heart failure, fatal arrhythmia, cardiac arrest because of other causes, cerebrovascular diseases, pulmonary embolism, peripheral artery diseases, and sudden otherwise unexplained death. Non-CV mortality was defined as all other causes of death, i.e., infection, malignancies, gastrointestinal hemorrhage, accidents, and miscellaneous. Allcause mortality included CV and non-CV death. Patients were censored at last follow-up, switched to another dialysis unit, or received a kidney transplant.

\subsection{Statistical Analysis}

The continuous variables were presented as mean \pm standard deviation, and the categorical variables were expressed as number (\%). The correlation coefficients between covariates of interest were calculated. The univariate Cox regression analysis was performed to investigate the independence of risk factors associated with all-cause and CV mortality. If hazard ratios (HRs) for the variables in the univariate analysis were significant, we would select the covariates into the multivariable regression model and GDF15-based death prediction model. Multivariable adjusted hazard ratios (aHRs) of different mortality risks were calculated for plasma GDF15 tertiles in the Cox regression model. The cumulative survival probability and proportional hazards were presented by graphical methods. To assess the predictive accuracy of GDF15 tertiles and GDF15-based risk score for mortality, the area under ROC curve (AUC) was used as the criterion [3]. An AUC of 0.5 indicates no predictive ability, whereas a value of 1 represents perfect predictive ability. A $p$-value $<0.05$ was considered statistically significant. We used the PASW Statistics SPSS version 22.0 (IBM, City, NY, USA) to analyze all bio-clinical data of MHD patients.

\section{Results}

\subsection{Primary Analyses: Evaluation of the Clinical Candidate Predictors}

The final study sample included 170 MHD patients with complete medical records and follow-up. Baseline bio-clinical data of the whole study population with comparisons 
between different concentration groups are summarized in Table 1. Plasma GDF15 levels were categorized into low levels (<1314.8 pg/mL), medium levels (1314.8-1707.1 pg/mL), and high levels (>1707.1 pg/mL) as the tertiles 1, 2, and 3, respectively. Age, prevalence of DM, prior history of CVD, HD vintage, and albumin levels were significantly different among three GDF15 tertiles. The mean age of low, medium, and high tertile was $59.1 \pm 6.8$, $60.3 \pm 8.1$, and $71.1 \pm 7.1$ years; namely, the older adults exhibit higher GDF15 levels. The prevalence of DM was $41.1 \%, 42.9 \%$, and $50.0 \%$ in tertiles 1, 2 and 3, respectively. Patients in tertile 3 had the highest prevalence of a prior CVD history $(62.5 \%)$. Patients in tertile 3 had the longest HD vintage $(80.9 \pm 34.2$ months). The mean albumin level in the tertiles 1,2 and 3 was $4.0 \pm 0.4,3.9 \pm 0.4$ and $3.8 \pm 0.5 \mathrm{~g} / \mathrm{dL}$, respectively. In the univariate Cox regression analysis of clinical prognostic factors (Table 2), older age, higher GDF15 and lower albumin levels were significantly associated with all-cause mortality (HR: 1.074 (95\% confidence interval (CI): 1.037-1.112), $p<0.01 ; 1.001$ (95\% CI: 1.000-1.001), $p<0.01 ; 0.200$ (95\% CI: $0.100-0.402), p<0.01$; respectively). Furthermore, the associations between age, higher GDF15, and lower albumin levels for CV mortality remained significant [HR: 1.086 (95\% CI: 1.040-1.133), $p<0.01 ; 1.001$ (95\% CI: 1.000-1.001), $p<0.01 ; 0.377$ (95\% CI: 0.160-0.884), $p<0.05$; respectively). Although the HD vintage was associated with all-cause mortality (HR: 1.006 (95\% CI: 1.001-1.011), $p<0.05)$, the association between HD vintage and CV mortality was insignificant [HR: 1.004 (95\% CI: 0.998-1.011), $p=0.17$ ]. Collectively, patients with older age, higher GDF15 and lower albumin levels are more likely to have greater risks in all-cause and CV mortality. Table 3 summarizes the multivariate Cox regression analysis of clinical candidate predictors for all-cause and CV mortality. Age, GDF15, and albumin were independently associated with overall mortality (aHR: 1.044 (95\% CI: 1.007-1.083), $p<0.05$; 1.001 (95\% CI: 1.000-1.001), $p<0.01 ; 0.281$ (95\% CI: 0.141-0.560), $p<0.01$; respectively).

Table 1. Age, diabetes mellitus, cardiovascular diseases, hemodialysis vintage, and albumin differ significantly in the comparisons of baseline bio-clinical characteristics according to GDF15 tertiles.

\begin{tabular}{|c|c|c|c|}
\hline Variables & $\begin{array}{c}\text { Tertile } 1 \\
<1314.8 \mathrm{pg} / \mathrm{mL}\end{array}$ & $\begin{array}{c}\text { Tertile } 2 \\
\text { 1314.8-1707.1 pg/mL }\end{array}$ & $\begin{array}{c}\text { Tertile } 3 \\
>\mathbf{1 7 0 7 . 1} \mathrm{pg} / \mathrm{mL}\end{array}$ \\
\hline Patients, $n(\%)$ & $56(32.9)$ & $56(32.9)$ & $58(41.4)$ \\
\hline Age (years) & $59.1 \pm 6.8$ & $60.3 \pm 8.1$ & $71.1 \pm 7.1$ \\
\hline Male, $n(\%)$ & $31(55.4)$ & $27(48.2)$ & $24(41.4)$ \\
\hline Diabetes mellitus, $n(\%)$ & $23(41.1)$ & $24(42.9)$ & $29(50.0)$ \\
\hline Cardiovascular diseases, $n(\%)$ & 80 (47.9) & $51(40.2)$ & $25(62.5)$ \\
\hline Hypertension, $n(\%)$ & $26(46.4)$ & $32(57.1)$ & $34(58.6)$ \\
\hline Systolic blood pressure (mmHg) & $135.3 \pm 21.4$ & $134.1 \pm 21.7$ & $142.3 \pm 22.7$ \\
\hline Diastolic blood pressure (mmHg) & $76.4 \pm 8.6$ & $78.3 \pm 11.4$ & $79.3 \pm 14.2$ \\
\hline Hemodialysis vintage (months) & $72.2 \pm 58.4$ & $62.5 \pm 51.8$ & $80.9 \pm 34.2$ \\
\hline nPCR (g/kg/day) & $1.2 \pm 0.3$ & $1.1 \pm 0.3$ & $1.1 \pm 0.3$ \\
\hline GDF15 (pg/mL) & $1046.6 \pm 203.0$ & $1525.4 \pm 112.7$ & $2298.6 \pm 637.9$ \\
\hline Albumin (g/dL) & $4.0 \pm 0.4$ & $3.9 \pm 0.4$ & $3.8 \pm 0.5$ \\
\hline Aspartate aminotransferase (IU/L) & $15.0 \pm 5.1$ & $15.5 \pm 6.8$ & $17.8 \pm 8.4$ \\
\hline Alanine aminotransferase (IU/L) & $13.2 \pm 8.3$ & $14.8 \pm 13.6$ & $16.6 \pm 13.0$ \\
\hline Total cholesterol (mg/dL) & $192.2 \pm 51.3$ & $184.3 \pm 47.6$ & $192.5 \pm 46.7$ \\
\hline Triglyceride (mg/dL) & $243.7 \pm 201.4$ & $194.4 \pm 172.1$ & $179.8 \pm 23.6$ \\
\hline Low-density lipoprotein & $108.1 \pm 39.4$ & $101.9 \pm 34.1$ & $111.8 \pm 33.5$ \\
\hline Blood urea nitrogen (mg/dL) & $55.9 \pm 17.8$ & $59.5 \pm 17.7$ & $61.8 \pm 20.0$ \\
\hline Creatinine $(\mathrm{mg} / \mathrm{dL})$ & $9.4 \pm 2.0$ & $10.0 \pm 1.9$ & $10.0 \pm 1.6$ \\
\hline Blood glucose (mg/dL) & $141.7 \pm 59.0$ & $143.3 \pm 65.0$ & $135.1 \pm 84.5$ \\
\hline Uric acid (mg/dL) & $7.4 \pm 1.4$ & $7.3 \pm 1.3$ & $7.2 \pm 1.1$ \\
\hline Potassium $\left(\mathrm{mmol} \mathrm{L}^{-1}\right)$ & $4.6 \pm 0.9$ & $4.6 \pm 0.9$ & $4.3 \pm 0.8$ \\
\hline Calcium (mg/dL) & $9.4 \pm 0.8$ & $9.3 \pm 0.8$ & $9.1 \pm 0.7$ \\
\hline Phosphate (mg/dL) & $4.6 \pm 1.7$ & $4.3 \pm 1.4$ & $5.0 \pm 1.4$ \\
\hline Calcium-phosphate product & $42.1 \pm 15.1$ & $40.2 \pm 12.9$ & $45.9 \pm 13.6$ \\
\hline
\end{tabular}


Table 1. Cont

\begin{tabular}{|c|c|c|c|}
\hline Variables & $\begin{array}{c}\text { Tertile } 1 \\
<1314.8 \mathrm{pg} / \mathrm{mL} \\
\end{array}$ & $\begin{array}{c}\text { Tertile } 2 \\
\text { 1314.8-1707.1 pg/mL }\end{array}$ & $\begin{array}{c}\text { Tertile } 3 \\
>\mathbf{1 7 0 7 . 1} \mathrm{pg} / \mathrm{mL}\end{array}$ \\
\hline Intact parathyroid hormone (pg/mL) & $164.1 \pm 220.5$ & $153.8 \pm 145.2$ & $184.9 \pm 200.2$ \\
\hline Hemoglobin $(\mathrm{g} / \mathrm{dL})$ & $10.8 \pm 1.3$ & $10.4 \pm 1.2$ & $10.6 \pm 1.3$ \\
\hline Hematocrit (\%) & $32.2 \pm 3.8$ & $31.1 \pm 3.5$ & $32.0 \pm 3.7$ \\
\hline Platelet $(\mathrm{k} / \mu \mathrm{L})$ & $209.3 \pm 66.3$ & $185.5 \pm 71.4$ & $199.9 \pm 56.1$ \\
\hline
\end{tabular}

Continuous variables were expressed as mean \pm SD. Categorical variables are expressed as $n$ (\%). Boldface indicates where the values differ significantly between GDF15 tertiles. GDF15 = growth differentiation factor-15. nPCR = normalized protein catabolic rate.

Table 2. Age, GDF15, and albumin as significant prognostic parameters for all-cause and CV mortality in the univariate Cox regression analysis.

\begin{tabular}{lllll}
\hline & All-Cause Mortality & & CV Mortality & \\
\hline Age & HR (95\% CI) & $p$-Value & HR $(95 \%$ CI $)$ & $p$-Value \\
Male & $\mathbf{1 . 0 7 4}(\mathbf{1 . 0 3 7 - 1 . 1 1 2 )}$ & $p<\mathbf{0 . 0 1}$ & $\mathbf{1 . 0 8 6} \mathbf{( 1 . 0 4 0 - 1 . 1 3 3 )}$ & $p<\mathbf{0 . 0 1}$ \\
HD vintage & $0.867(0.476-1.578)$ & $p=0.64$ & $1.139(0.556-2.335)$ & $p=0.72$ \\
GDF15 & $1.006(1.001-1.011)$ & $p<0.05$ & $1.004(0.998-1.011)$ & $p=0.17$ \\
SBP & $\mathbf{1 . 0 0 1}(\mathbf{1 . 0 0 0 - 1 . 0 0 1 )}$ & $p<\mathbf{0 . 0 1}$ & $\mathbf{1 . 0 0 1}(\mathbf{1 . 0 0 0 - 1 . 0 0 1 )}$ & $p<\mathbf{0 . 0 1}$ \\
DBP & $1.012(0.998-1.026)$ & $p=0.08$ & $1.021(1.005-1.038)$ & $p<0.05$ \\
Blood glucose & $0.981(0.998-1.026)$ & $p=0.16$ & $0.982(0.952-1.014)$ & $p=0.27$ \\
nPCR & $1.001(0.996-1.006)$ & $p=0.72$ & $1.003(0.998-1.008)$ & $p=0.30$ \\
Albumin & $0.840(0.284-2.488)$ & $p=0.75$ & $0.875(0.239-3.200)$ & $p=0.84$ \\
AST & $\mathbf{0 . 2 0 0 ( 0 . 1 0 0 - 0 . 4 0 2 )}$ & $p<\mathbf{0 . 0 1}$ & $\mathbf{0 . 3 7 7}(\mathbf{0 . 1 6 0 - 0 . 8 8 4 )}$ & $p<\mathbf{0 . 0 5}$ \\
ALT & $0.983(0.938-1.030)$ & $p=0.47$ & $0.957(0.898-1.019)$ & $p=0.17$ \\
T-Cholesterol & $1.014(0.993-1.036)$ & $p=0.19$ & $1.018(0.994-1.043)$ & $p=0.14$ \\
Triglyceride & $0.999(0.992-1.005)$ & $p=0.65$ & $1.000(0.993-1.008)$ & $p=0.90$ \\
LDL & $0.998(0.996-1.001)$ & $p=0.15$ & $0.998(0.995-1.001)$ & $p=0.19$ \\
BUN & $0.998(0.990-1.006)$ & $p=0.63$ & $1.001(0.991-1.011)$ & $p=0.83$ \\
Creatinine & $1.008(0.992-1.023)$ & $p=0.34$ & $1.009(0.991-1.028)$ & $p=0.34$ \\
Uric acid & $1.029(0.879-1.204)$ & $p=0.73$ & $1.113(0.920-1.347)$ & $p=0.27$ \\
Potassium & $1.077(0.859-1.350)$ & $p=0.52$ & $1.088(0.830-1.426)$ & $p=0.54$ \\
Calcium & $0.788(0.552-1.125)$ & $p=0.19$ & $0.773(0.504-1.185)$ & $p=0.24$ \\
Phosphate & $0.795(0.515-1.229)$ & $p=0.30$ & $0.602(0.347-1.045)$ & $p=0.07$ \\
Ca-P product & $1.075(0.897-1.288)$ & $p=0.44$ & $1.038(0.829-1.300)$ & $p=0.75$ \\
iPTH & $1.005(0.984-1.026)$ & $p=0.64$ & $0.999(0.973-1.025)$ & $p=0.92$ \\
Hemoglobin & $1.001(1.000-1.002)$ & $p=0.07$ & $1.001(0.999-1.002)$ & $p=0.43$ \\
Hematocrit & $1.066(0.818-1.390)$ & $p=0.63$ & $1.013(0.739-1.389)$ & $p=0.94$ \\
Platelet & $1.032(0.939-1.133)$ & $p=0.51$ & $1.017(0.910-1.137)$ & $p=0.77$ \\
Boldfat & $1.002(0.998-1.007)$ & $p=0.35$ & $1.005(0.997-1.010)$ & $p=0.09$ \\
\hline
\end{tabular}

Boldface indicates significant predictors for both all-cause and CV mortality. ALT $=$ Alanine aminotransferase; AST $=$ aspartate aminotransferase; BUN = blood urea nitrogen; Ca- $p=$ Calcium-phosphate; $\mathrm{CV}=$ cardiovascular; CVD = cardiovascular diseases; $\mathrm{CI}=$ confidence interval; GDF15 = growth differentiation factor-15; $\mathrm{HD}=$ Hemodialysis; HR, hazard ratio; iPTH = intact parathyroid hormone; LDL = Low-density lipoprotein; $\mathrm{nPCR}=$ normalized protein catabolic rate; $\mathrm{SBP}=$ Systolic blood pressure; $\mathrm{T}$-Cholesterol $=$ Total Cholesterol .

Table 3. Age, GDF15, and albumin as significant prognostic parameters for all-cause mortality in the multivariate Cox regression analysis.

\begin{tabular}{lllll}
\hline \multicolumn{4}{l}{ All-Cause Mortality } & \multicolumn{3}{l}{ CV Mortality } & \\
\hline HR $(95 \% \mathrm{CI})$ & $p$-Value & HR $(95 \% \mathrm{CI})$ & $p$-Value \\
\hline Age & $1.044(1.007-1.083)$ & $p<0.05$ & $1.062(1.015-1.112)$ & $p<0.01$ \\
GDF15 & $1.001(1.000-1.001)$ & $p<0.01$ & $1.001(1.000-1.001)$ & $p<0.05$ \\
Albumin & $0.281(0.141-0.560)$ & $p<0.01$ & $0.550(0.235-1.289)$ & $p=0.17$ \\
\hline
\end{tabular}

$\overline{\mathrm{CVD}}=$ cardiovascular diseases; $\mathrm{CI}=$ confidence interval; GDF15 = growth differentiation factor-15. 
Figure 1 illustrates cumulative survival curves of mortality risks among patients with three GDF15 concentration tertiles after adjusting for age and albumin. The highest tertile of GDF15 is associated with an incremental risk of all-cause mortality (aHR: 3.06 [95\% CI: 1.20-7.82], $p<0.05$ ). The association between the highest tertile of GDF15 and CV mortality remains robust after multivariable adjustment (aHR: 3.11 [95\% CI: 1.02-9.50], $p<0.05$ ). Figure 2 demonstrated the ROC analysis using GDF15 tertiles as a predictor of mortality in the whole study population following up for over 30 months. The AUC provided by the tertiles of GDF15 for all-cause and CV mortality is $0.681(95 \% \mathrm{CI}=0.589$ to $0.774, p<0.01)$ and $0.684(95 \% \mathrm{CI}=0.577$ to $0.791, p<0.01)$, respectively. The ROC analysis using age as a predictor of overall mortality is demonstrated in the Figure 1. The AUC provided by older age for overall mortality is $0.687(95 \% \mathrm{CI}=0.594$ to $0.779, p<0.01)$. The cut-off value for age is 65 years old. The ROC analysis using the lower albumin level as a predictor of overall mortality is demonstrated in the Figure 2. The AUC provided by the lower albumin level for overall mortality is $0.691(95 \% \mathrm{CI}=0.600$ to $0.782, p<0.01)$. The cut-off value for the albumin level is $3.8 \mathrm{~g} / \mathrm{dL}$. As a result, age $>65$ years old (score 1), GDF15 tertiles 1-3 (score 1-3) and hypoalbuminemia $<3.8 \mathrm{~g} / \mathrm{dL}$ (score 1) were combined as a death score system in the secondary analyses.

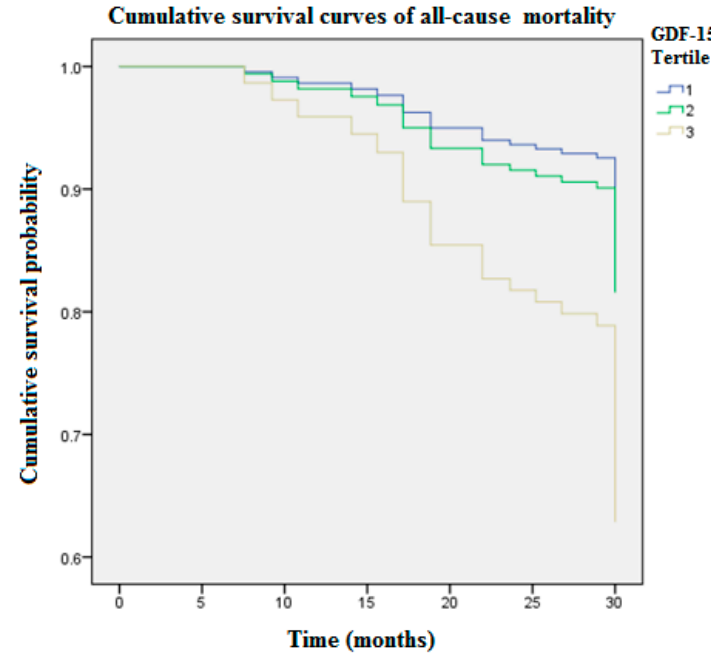

(A)

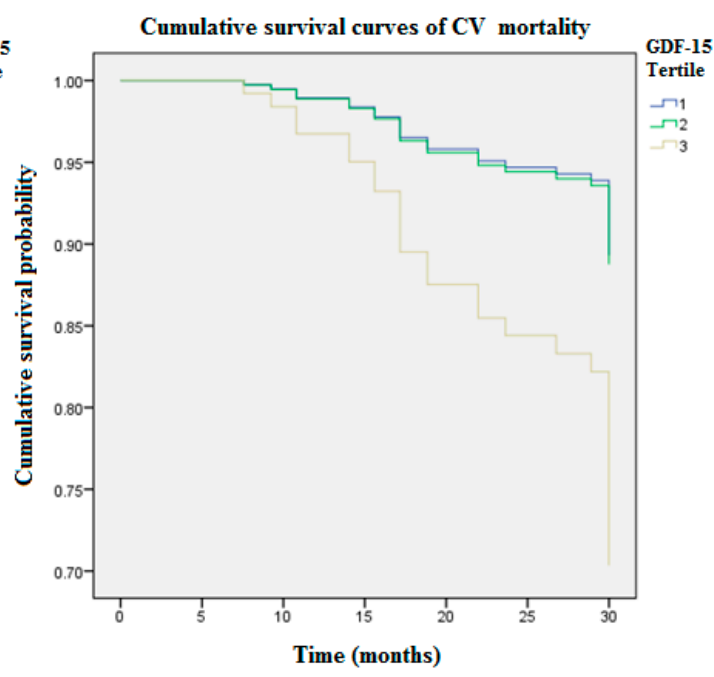

(B)

Figure 1. The cumulative survival curves of mortality risks showed GDF15 tertiles remained significant after adjusting for age and albumin during 4650.9 person-months follow-up (Tertile 1 GDF15 $<1314.8$ pg/mL; Tertile 2 GDF15 = 1314.8-1707.1 pg/mL; Tertile 3 GDF15 > $1707.1 \mathrm{pg} / \mathrm{mL})$. (A) The highest tertile of GDF15 (>1707.1 pg/mL) was associated with an incremental risk of all-cause mortality (aHR: 3.06 [95\% CI: 1.20-7.82], $p<0.05$ ). (B) The association between the highest tertile of GDF15 and CV mortality remained robust after multivariable adjustment (aHR: 3.11 [95\% CI: 1.02-9.50], $p<0.05)$. GDF15 = growth differentiation factor-15. 


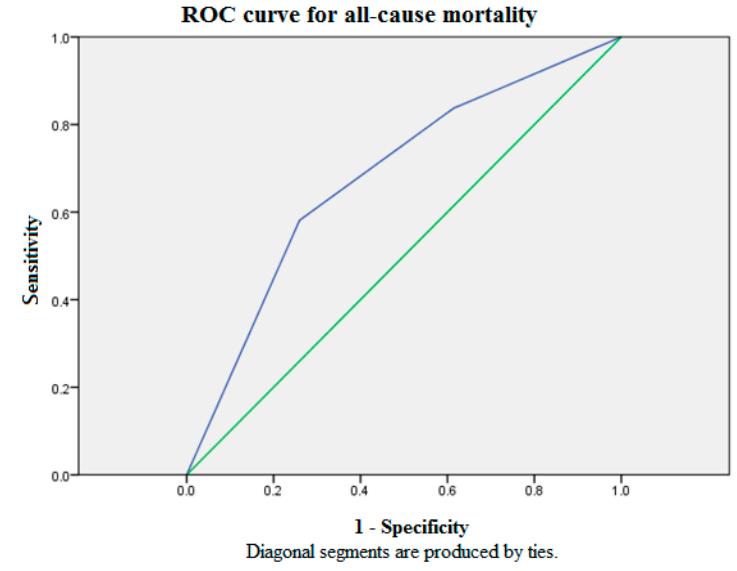

(A)

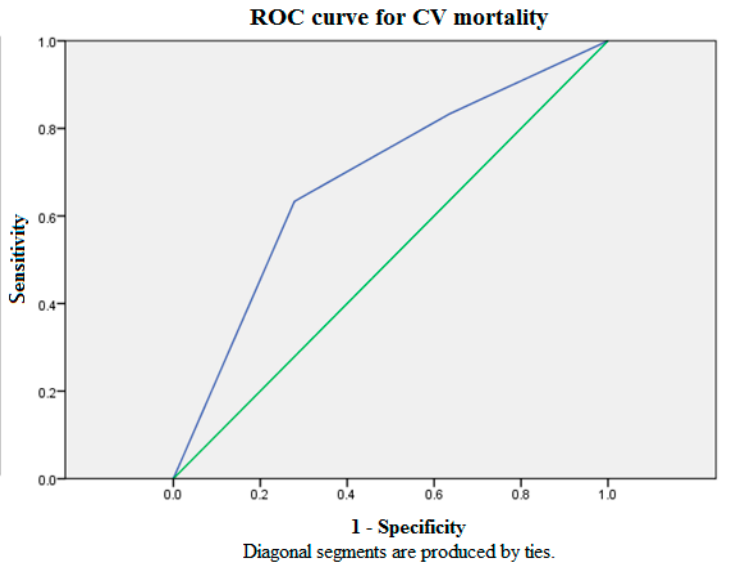

(B)

Figure 2. The ROC analysis of GDF15 tertiles for mortality showed suboptimal discriminatory power in the whole study population over 30 months of follow-up. (A) The AUC provided by GDF15 tertiles for all-cause mortality was 0.681 (95\% CI $=0.589$ to $0.774, p<0.01)$. (B) The AUC provided by GDF15 tertiles for CV mortality was $0.684(95 \% \mathrm{CI}=0.577$ to $0.791, p<0.01)$. Source of the curve: green line $=$ GDF15 tertiles; blue line $=$ reference. AUC = area under curve; $\mathrm{CV}=$ cardiovascular; GDF15 = growth differentiation factor-15; ROC = receiver operating characteristics.

\subsection{Secondary Analyses: Evaluation of the GDF15-Based Death Prediction Model}

Table 4 summarizes the bio-clinical data and fatal events according to our GDF15based risk score system. Three GDF15 concentration tertiles are designated as scores 1, 2 and 3. Scores 1 or 0 of the clinical predictors are defined by the cut-off value of the ROC analysis. All patients are stratified by the GDF15-based risk score system into new categories. Figure 3 shows the comparison of ROC analysis between GDF15 tertiles and the GDF15-based risk score model for mortality in the whole study population over 30 months of follow-up. The AUC provided by the GDF15-based risk score model for all-cause mortality is $0.745(95 \% \mathrm{CI}=0.666$ to 0.824$)$, and $\mathrm{CV}$ mortality $0.715(95 \% \mathrm{CI}=0.625$ to 0.805$)$. Consideration of GDF15 concentration in the risk factor analysis increases the sensitivity and the specificity for all-cause and CV mortality. In conclusion, our GDF15-based risk score system may serve as an early warning for timely and appropriate intervention of clinical events in the MHD population.

Table 4. Comparison of bio-clinical parameters and fatal events according to GDF15-based risk score system in the whole study population.

\begin{tabular}{cccccc}
\hline & Score $=\mathbf{1}$ & Score $=\mathbf{2}$ & Score $=\mathbf{3}$ & Score $=\mathbf{4}$ & Score $=\mathbf{5}$ \\
\hline Patients, $n(\%)$ & $32(18.8)$ & $55(32.4)$ & $25(14.7)$ & $39(22.9)$ & $19(11.2)$ \\
\hline All-cause death, $n(\%)$ & $0(0)$ & $10(18.2)$ & $9(36.0)$ & $12(30.8)$ & $12(63.2)$ \\
CV death, $n(\%)$ & $0(0)$ & $7(12.7)$ & $6(24.0)$ & $10(25.6)$ & $7(36.8)$ \\
Age (years) & $56.3 \pm 4.0$ & $58.9 \pm 6.4$ & $61.6 \pm 7.1$ & $71.2 \pm 6.6$ & $74.2 \pm 4.7$ \\
Male, $n$ (\%) & $12(37.5)$ & $33(60.0)$ & $15(60.0)$ & $13(33.3)$ & $9(47.4)$ \\
DM, $n(\%)$ & $9(28.1)$ & $24(43.6)$ & $12(48.0)$ & $20(51.3)$ & $11(57.9)$ \\
CVD, $n(\%)$ & $12(37.5)$ & $22(40.0)$ & $11(44.0)$ & $24(61.5)$ & $10(52.6)$ \\
Hypertension, $n(\%)$ & $19(59.4)$ & $29(52.7)$ & $13(52.0)$ & $21(53.8)$ & $10(52.6)$ \\
SBP $(\mathrm{mmHg})$ & $136.4 \pm 16.4$ & $134.3 \pm 23.2$ & $135.0 \pm 25.5$ & $141.1 \pm 21.8$ & $143.8 \pm 23.7$ \\
DBP $(\mathrm{mmHg})$ & $79.1 \pm 6.9$ & $79.0 \pm 10.7$ & $71.2 \pm 11.2$ & $79.7 \pm 14.4$ & $79.0 \pm 13.1$ \\
HD vintage $(\mathrm{months})$ & $72.9 \pm 49.2$ & $59.6 \pm 51.9$ & $81.9 \pm 61.6$ & $83.1 \pm 41.5$ & $70.4 \pm 32.4$ \\
nPCR $(\mathrm{g} / \mathrm{kg} / \mathrm{day})$ & $1.2 \pm 0.3$ & $1.1 \pm 0.3$ & $1.1 \pm 0.3$ & $1.0 \pm 0.2$ & $1.1 \pm 0.4$ \\
GDF15 $(\mathrm{pg} / \mathrm{mL})$ & $1098.3 \pm 181.3$ & $1303.8 \pm 324.3$ & $1731.8 \pm 638.1$ & $2157.2 \pm 633.8$ & $2266.7 \pm 592.8$ \\
Albumin $(\mathrm{g} / \mathrm{dL})$ & $4.1 \pm 0.3$ & $4.0 \pm 0.4$ & $3.8 \pm 0.4$ & $3.8 \pm 0.4$ & $3.3 \pm 0.4$ \\
\hline
\end{tabular}


Table 4. Cont.

\begin{tabular}{|c|c|c|c|c|c|}
\hline & Score $=1$ & Score $=2$ & Score $=3$ & Score $=4$ & Score $=5$ \\
\hline AST (IU/L) & $15.1 \pm 5.0$ & $14.7 \pm 5.5$ & $15.7 \pm 6.9$ & $18.9 \pm 9.1$ & $17.3 \pm 8.0$ \\
\hline ALT (IU/L) & $14.9 \pm 8.4$ & $14.1 \pm 13.5$ & $13.2 \pm 10.6$ & $14.8 \pm 11.0$ & $19.3 \pm 15.3$ \\
\hline T-Cholesterol (mg/dL) & $188.5 \pm 48.5$ & $190.3 \pm 51.3$ & $186.6 \pm 43.1$ & $199.4 \pm 52.9$ & $186.0 \pm 41.6$ \\
\hline Triglyceride $(\mathrm{mg} / \mathrm{dL})$ & $225.7 \pm 180.7$ & $222.7 \pm 203.9$ & $198.5 \pm 165.0$ & $202.1 \pm 197.5$ & $172.6 \pm 141.6$ \\
\hline LDL & $107.2 \pm 41.4$ & $105.8 \pm 36.3$ & $103.0 \pm 29.8$ & $114.4 \pm 37.4$ & $103.5 \pm 28.5$ \\
\hline BUN (mg/dL) & $53.5 \pm 16.1$ & $57.2 \pm 17.1$ & $65.1 \pm 16.9$ & $59.4 \pm 18.0$ & $65.5 \pm 25.8$ \\
\hline Creatinine $(\mathrm{mg} / \mathrm{dL})$ & $9.6 \pm 2.0$ & $9.8 \pm 2.2$ & $10.5 \pm 1.4$ & $9.5 \pm 1.5$ & $9.9 \pm 1.7$ \\
\hline Blood glucose (mg/dL) & $122.0 \pm 40.2$ & $130.9 \pm 57.5$ & $152.6 \pm 63.2$ & $133.6 \pm 70.2$ & $137.0 \pm 69.7$ \\
\hline Uric acid $(\mathrm{mg} / \mathrm{dL})$ & $7.5 \pm 1.3$ & $7.3 \pm 1.5$ & $7.6 \pm 1.1$ & $6.9 \pm 1.1$ & $7.2 \pm 0.9$ \\
\hline Potassium $\left(\mathrm{mmol} \mathrm{L}^{-1}\right)$ & $4.6 \pm 1.0$ & $4.5 \pm 0.9$ & $4.8 \pm 0.7$ & $4.2 \pm 0.6$ & $4.1 \pm 1.0$ \\
\hline Calcium (mg/dL) & $9.3 \pm 0.7$ & $9.2 \pm 0.7$ & $9.4 \pm 0.8$ & $9.2 \pm 0.7$ & $9.1 \pm 0.7$ \\
\hline Phosphate (mg/dL) & $4.5 \pm 1.5$ & $4.4 \pm 1.7$ & $5.1 \pm 1.5$ & $4.7 \pm 1.2$ & $5.0 \pm 1.7$ \\
\hline Ca-P product & $41.6 \pm 14.0$ & $40.9 \pm 15.2$ & $47.0 \pm 14.4$ & $42.8 \pm 10.4$ & $44.5 \pm 16.2$ \\
\hline iPTH (pg/mL) & $213.9 \pm 254.0$ & $177.5 \pm 195.8$ & $328.8 \pm 320.2$ & $236.4 \pm 230.5$ & $198.4 \pm 214.2$ \\
\hline Hemoglobin (g/dL) & $10.8 \pm 1.3$ & $10.6 \pm 1.3$ & $10.7 \pm 1.1$ & $10.6 \pm 1.2$ & $10.4 \pm 1.6$ \\
\hline Hematocrit (\%) & $32.0 \pm 3.7$ & $31.4 \pm 3.7$ & $31.9 \pm 3.2$ & $31.9 \pm 3.5$ & $31.7 \pm 4.5$ \\
\hline Platelet $(\mathrm{k} / \mu \mathrm{L})$ & $205.2 \pm 62.6$ & $200.0 \pm 68.2$ & $192.1 \pm 78.7$ & $190.4 \pm 57.9$ & $203.7 \pm 64.1$ \\
\hline
\end{tabular}

Continuous variables were expressed as mean $\pm \mathrm{SD}$. Categorical variables are expressed as $n(\%)$. ALT $=$ Alanine aminotransferase; AST = aspartate aminotransferase; $\mathrm{BUN}=$ blood urea nitrogen; $\mathrm{Ca}-\mathrm{P}=$ Calcium-phosphate $\mathrm{CV}=$ cardiovascular; $\mathrm{CVD}=$ cardiovascular diseases; DBP = Diastolic blood pressure; DM = Diabetes mellitus; GDF15 = growth differentiation factor-15; HD = Hemodialysis; $\mathrm{iPTH}=$ intact parathyroid hormone; $\mathrm{LDL}=$ Low-density lipoprotein; $\mathrm{nPCR}=$ normalized protein catabolic rate; $\mathrm{SBP}=\mathrm{Systolic}$ blood pressure; $\mathrm{T}$-Cholesterol $=$ Total Cholesterol.

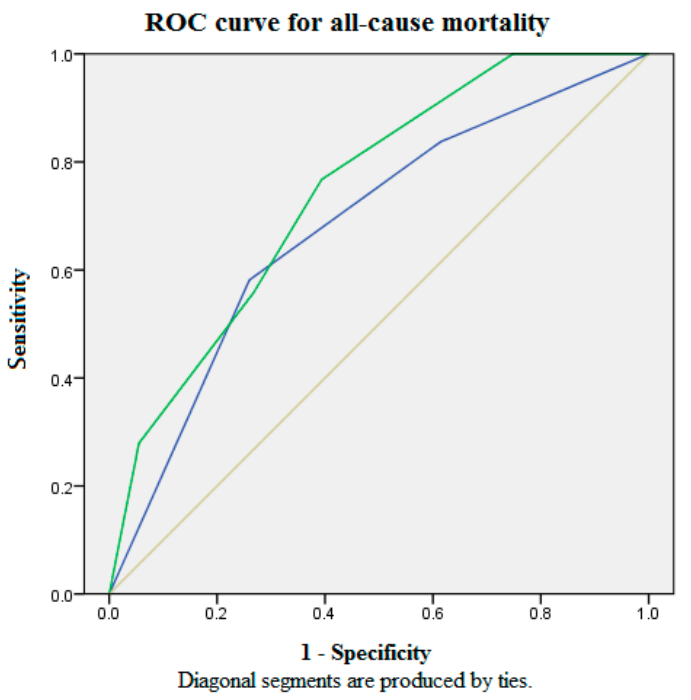

(A)

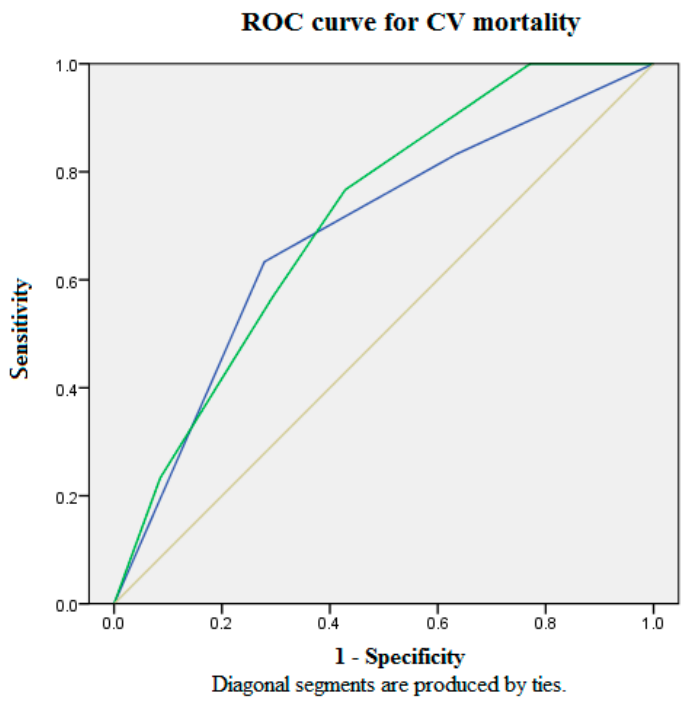

(B)

Figure 3. The ROC analysis of the GDF15-based risk score model showed optimal discriminatory power with comparison to GDF15 tertiles in the whole study population over 30 months of follow-up. (A) The AUC provided by GDF15 tertiles and the GDF15-based risk score model for all-cause mortality was $0.681(95 \% \mathrm{CI}=0.589$ to 0.774$)$ and $0.745(95 \% \mathrm{CI}=0.666$ to 0.824$)$, respectively. (B) The AUC provided by GDF15 tertiles and the GDF15-based risk score model for CV mortality was 0.684 (95\% CI $=0.577$ to 0.791$)$ and 0.715 (95\% CI = 0.625 to 0.805$)$, respectively. Source of the curve: green line $=$ GDF15-based risk score model; blue line $=$ GDF15 tertiles; yellow line = reference. AUC = area under curve; CV = cardiovascular; GDF15 = growth differentiation factor-15; ROC = receiver operating characteristics.

\section{Discussion}

The optimal medical services to improve the patient safety are delivery of early detection and sustained monitoring of high-risk subjects. To deploy a timely and effective therapeutic strategy in preparation, a robust surrogate biomarker to warn clinicians in 
advance is crucial for the CKD population because of high prevalence of morbidity and mortality. Especially in MHD patients prone to sudden cardiac death due to persistent inflammation accompanied by malnutrition, the precise prognostic evaluation and therapeutic decision-making are particularly important [15]. Considering their underlying multiple comorbidities, it seems unlikely that a single biomarker assessment is sufficient to understand patients' overall burden of ailments. Hijazi et al. consolidated age, biomarkers, and clinical parameters as a novel $\mathrm{ABC}$-death risk score in the high-risk CV population [17]. Based on their ABC-death risk model, all clinical candidate predictors in our MHD patients were evaluated using the Cox regression analysis for all-cause and CV mortality in the first step (Table 2). Our results demonstrate MHD patients with older age, higher plasma GDF15 concentrations, and lower albumin levels are more likely to have greater risks for all-cause death (HR: 1.074 ((95\% CI: 1.037-1.112), 1.001 ((95\% CI: 1.000-1.001), 0.200 (95\% CI: 0.100-0.402), respectively) but also CV mortality (HR: 1.086 (95\% CI: 1.040-1.133), 1.001 (95\% CI: 1.000-1.001), 0.377 (95\% CI: 0.160-0.884), respectively). HD vintage lost the predictive power for CV mortality (Table 2), despite being associated with all-cause death and significantly different among three groups of GDF15 tertiles (Table 1). In the multivariate Cox regression analysis for clinical candidate predictors (Table 3), age, GDF15, and albumin were independently associated with overall mortality (aHR: 1.044 (95\% CI: 1.007-1.083), 1.001 (95\% CI: 1.000-1.001), 0.281 (95\% CI: 0.141-0.560), respectively). Accordingly, older age, higher GDF15, and hypoalbuminemia were integrated as a novel death risk score system in our modified prediction model. After adjusting for age and albumin in the cumulative survival curve analysis with respect to different GDF15 tertiles among the whole study population (Figure 1), the highest tertile of GDF15 $(>1707.1 \mathrm{pg} / \mathrm{mL}$ ) was associated with an incremental risk for not only all-cause death (aHR: 3.06 (95\% CI: 1.20-7.82), $p<0.05$ ) but also CV mortality (aHR: 3.11 (95\% CI: 1.02-9.50), $p<0.05$ ). In the ROC analysis for mortality over 4650.9 person-months follow-up, however, the AUC provided by GDF15 tertiles for all-cause and CV mortality was $0.681(95 \% \mathrm{CI}=0.589$ to $0.774, p<0.01)$ and $0.684(95 \% \mathrm{CI}=0.577$ to $0.791, p<0.01)$, respectively. The prediction model using GDF15 tertiles has inadequate discriminatory power (Figure 2). Likewise, the AUC provided by older age ( $>65$ years old) for overall mortality is $0.687(95 \% \mathrm{CI}=0.594$ to $0.779, p<0.01)$ (Figure 1). The AUC provided by the lower albumin level $(<3.8 \mathrm{~g} / \mathrm{dL})$ for overall mortality is $0.691(95 \% \mathrm{CI}=0.600$ to $0.782, p<0.01)$ (Figure 2). The above results inspire us to combine age, GDF15, and hypoalbuminemia as a brand-new risk score system to predict fatal events.

In the secondary analyses, age, biomarker GDF15, and hypoalbuminemia were incorporated into a novel GDF15-baesd death prediction model for the ROC analysis. As expected, our GDF15-based risk score is a more comprehensive system that provides stronger predictive values for both all-cause and CV mortality. The comparison of ROC analyses between GDF15 tertiles and the GDF15-based risk score model for mortality was provided in Figure 3. The AUC provided by the GDF15-based risk score model for all-cause and CV mortality was $0.745(95 \% \mathrm{CI}=0.666$ to 0.824$)$ and $0.715(95 \% \mathrm{CI}=0.625$ to 0.805), respectively.

GDF15 is reminiscent of a robust prognostic biomarker in the high-risk patients with CVD, including fatal arrhythmia, myocardial infarction and heart failure [17-20]. In addition to the predictive role of cardiac biomarker, increased GDF15 levels have been known to be associated with a greater risk of all-cause mortality [21]. However, there are few studies linking GDF15 to the CKD-related outcome-prediction models. Previous studies demonstrated that a higher circulating level of GDF15 was an independent predictor of CKD mortality after multivariate adjustment, and correlated with the severity of atherosclerosis, hypoalbuminemia, inflammation, HD vintage, and protein-energy wasting [22,23]. Nair et al. found that plasma GDF15 levels were correlated with intrarenal GDF15 expression in the tubulo-interstitial compartment and significantly associated with a decline in renal function or progression to end stage renal disease. Their research team concluded that GDF15 was not only a CKD outcome predictor, but rather directly involved in the intrarenal 
signaling pathways contributing to CKD progression [6]. Bansal et al. analyzed the baseline characteristics of 3664 CKD patients and found that those with higher plasma GDF15 concentrations had older age, the higher prevalence of DM and CVD history, and higher risks in CKD progression [8]. The results above support our findings that higher GDF15 levels were associated older age, longer HD duration, hypoalbuminemia, and higher risks in the fatal events. We acknowledge that the reliability of our ABC-death score model might be insufficient in relation to the relatively small sample size; however, such ABC-death prediction model was validated in a large sample study of 14,611 patients [12]. Furthermore, You et al. measured GDF15 using the same method and enzyme-linked immunosorbent assay kits (R\&D Systems, Minneapolis, MN, USA) as we used [10]. Their main result is similar to ours in which GDF15 is associated with all-cause mortality using adjusted death HRs. Both their and our study subjects are hemodialysis patients, suggesting that the patient heterogeneity may not exert a significant influence. Considering the nature of GDF15 is a stress-induced protein, elevated circulating GDF15 levels indeed assisted physicians in identifying the patients at a high risk for a myriad of adverse clinical outcomes.

Our study contributes differently from other mortality risk indicators based on a previously validated ABC-death risk score model in a large sample size of 14,611 highrisk CV patients [12]. We confirmed the external validity and generalizability of the ABC-death prediction model in the MHD population. Furthermore, we proved that a combination scoring system provides more robust predictive powers than a single mortality risk indicator. Considering the multiple comorbidities in patients with organ failure, it seems unlikely that a single biomarker assessment could comprehensively evaluate the patients' overall disease burden. Thus, we improve the insufficiency of single mortality risk indicator. In light of this, our risk scoring system could be applied in the monthly routine assessment of MHD patients. An elevated GDF15-based risk score warns clinicians to determine an appropriate intervention in advance.

The interest is increasing rapidly in the application of risk score systems and artificial intelligence (AI)-based decision tree assisted technologies to monitor the patient safety $[14,15]$. In recent years, the accessibility of electronic medical records for big data in the healthcare and development of machine learning to AI has grown tremendously. Using machine-learning algorithms to analyze a huge amount of clinical data/risk factors in the patient outcome prediction has been demonstrated to be effective $[23,24]$ and, in some case, even surpassing the performance of clinicians [25,26]. Our GDF15-based risk score system is designed based on the statistic regression analysis of clinical parameters and a robust biomarker to predict the mortality of CKD patients, providing a data bank in AI development. With the calibration settings of AI algorithms and extensive validation in the future, we hope this model can be implemented in clinical practice to alarm clinicians and patients for deploying a therapeutic strategy and decision-making in advance.

Several limitations of this study are noteworthy. Firstly, our study sample size was relatively small and the majority of study participants were Asian. Thus, our model applied to other populations should be interpreted with caution. Secondly, cross-sectional laboratory values and GDF15 levels might not reflect substantial intra-individual variability over time. Finally, all cases of sudden death and CV events were combined as CV mortality because we lacked sufficient power to examine specific events. We acknowledge that the pathophysiology of underlying CVD for various outcomes may differ.

\section{Conclusions}

Since the risk stratification alerts clinicians to deploy a differential diagnosis and corresponding treatment in advance, searching for a robust biomarker-based risk prediction model is of prime importance for MHD patients with extremely high mortality rates. Age, GDF15, and hypoalbuminemia predict all-cause and CV death in MHD patients, yet a combination scoring system provides more robust predictive powers. Elevated GDF15based risk score serves as a warning sign, indicating an urgent need for the further study to 
determine an appropriate intervention. In light of this, the GDF15-based death prediction model should be developed in the AI-based precision medicine.

$\begin{array}{ll}\begin{array}{ll}\text { Abbreviation } \\ \text { aHRs }\end{array} & \text { adjusted hazard ratios } \\ \text { AI } & \text { artificial intelligence } \\ \text { ALT } & \text { Alanine aminotransferase } \\ \text { AST } & \text { aspartate aminotransferase } \\ \text { AUC } & \text { area under ROC curve } \\ \text { BUN } & \text { blood urea nitrogen } \\ \text { CI } & \text { confidence interval } \\ \text { CKD } & \text { chronic kidney disease } \\ \text { Ca-P } & \text { Calcium-phosphate } \\ \text { CV } & \text { cardiovascular } \\ \text { CVD } & \text { cardiovascular diseases } \\ \text { DBP } & \text { Diastolic blood pressure } \\ \text { DM } & \text { Diabetes mellitus } \\ \text { GDF15 } & \text { growth differentiation factor-15 } \\ \text { HD } & \text { Hemodialysis } \\ \text { iPTH } & \text { intact parathyroid hormone } \\ \text { LDL } & \text { Low-density lipoprotein } \\ \text { MHD } & \text { maintenance hemodialysis } \\ \text { nPCR } & \text { normalized protein catabolic rate } \\ \text { ROC } & \text { receiver operating characteristic } \\ \text { SBP } & \text { Systolic blood pressure } \\ \text { T-Cholesterol } & \text { Total Cholesterol }\end{array}$

Author Contributions: J.-C.L. and J.-F.C. were responsible for study concept and design, interpretation of data, writing of the manuscript, and drafting of the manuscript. C.-Y.H. assisted in biochemical analysis and experimental supports of detection of biomarkers. J.-C.L. and P.-C.C. assisted with statistical analysis. J.-C.L. implemented the manuscript revision. All authors have read and agreed to the published version of the manuscript.

Funding: This research was funded by MOST 109-2628-B-385-001; Renal Care Joint Foundation; MOST 109-2221-E-038 -013, DP2-109-21121-01-O-01-03, DP2-108-21121-01-O-05-04, MOST-109-2918-I038-002, and TMU-NTUST-109-10).

Institutional Review Board Statement: The study was conducted according to the guidelines of the Declaration of Helsinki, and approved by the Institutional Review Board of En Chu Kong Hospital (ECKIRB1070102).

Informed Consent Statement: Informed consent was obtained from all subjects involved in the study.

Data Availability Statement: The numeric data used to support the findings of this study are available from the corresponding author, Jian-Chiun Liou, upon reasonable request.

Acknowledgments: We would like to thank Min-Jie Tsai for the proofreading and Renalysis Medical Care Co., Ltd. for clinical assistance.

Conflicts of Interest: The authors declare no conflict of interest.

\section{References}

1. Ko, W.-C.; Choy, C.-S.; Lin, W.-N.; Chang, S.-W.; Liou, J.-C.; Tung, T.-H.; Hsieh, C.-Y.; Chang, J.-F. Galectin-3 Interacts with Vascular Cell Adhesion Molecule-1 to Increase Cardiovascular Mortality in Hemodialysis Patients. J. Clin. Med. 2018, 7, 300. [CrossRef] [PubMed]

2. Mihai, S.; Codrici, E.; Popescu, I.D.; Enciu, A.-M.; Albulescu, L.; Necula, L.G.; Mambet, C.; Anton, G.; Tanase, C. InflammationRelated Mechanisms in Chronic Kidney Disease Prediction, Progression, and Outcome. J. Immunol. Res. 2018, 2018 , 2180373. [CrossRef]

3. Chang, J.-F.; Chou, Y.-S.; Wu, C.-C.; Chen, P.-C.; Ko, W.-C.; Liou, J.-C.; Hsieh, C.-Y.; Lin, W.-N.; Wen, L.-L.; Chang, S.-W.; et al. A Joint Evaluation of Neurohormone Vasopressin-Neurophysin II-Copeptin and Aortic Arch Calcification on Mortality Risks in Hemodialysis Patients. Front. Med. 2020, 7, 102. [CrossRef] [PubMed] 
4. Jehn, U.; Schütte-Nütgen, K.; Henke, U.; Bautz, J.; Pavenstädt, H.; Suwelack, B.; Reuter, S. Prognostic Value of Growth Differentiation Factor 15 in Kidney Donors and Recipients. J. Clin. Med. 2020, 9, 1333. [CrossRef]

5. Wischhusen, J.; Melero, I.; Fridman, W.H. Growth/Differentiation Factor-15 (GDF-15): From Biomarker to Novel Targetable Immune Checkpoint. Front. Immunol. 2020, 11, 951. [CrossRef]

6. Nair, V.; Robinson-Cohen, C.; Smith, M.R.; Bellovich, K.A.; Bhat, Z.Y.; Bobadilla, M.; Brosius, F.; de Boer, I.H.; Essioux, L.; Formentini, I.; et al. Growth Differentiation Factor-15 and Risk of CKD Progression. J. Am. Soc. Nephrol. 2017, 28, 2233-2240. [CrossRef]

7. Tuegel, C.; Katz, R.; Alam, M.; Bhat, Z.; Bellovich, K.; de Boer, I.; Brosius, F.; Gadegbeku, C.; Gipson, D.; Hawkins, J.; et al. GDF-15, Galectin 3, Soluble ST2, and Risk of Mortality and Cardiovascular Events in CKD. Am. J. Kidney Dis. 2018, 72, 519-528. [CrossRef]

8. Bansal, N.; Zelnick, L.; Shlipak, M.G.; Anderson, A.; Christenson, R.; Deo, R.; deFilippi, C.; Feldman, H.; Lash, J.; He, J.; et al. Cardiac and Stress Biomarkers and Chronic Kidney Disease Progression: The CRIC Study. Clin. Chem. 2019, 65, $1448-1457$. [CrossRef]

9. Stein, N.R.; Zelnick, L.R.; Anderson, A.H.; Christenson, R.H.; deFilippi, C.R.; Deo, R.; Go, A.S.; He, J.; Ky, B.; Lash, J.P.; et al. Associations Between Cardiac Biomarkers and Cardiac Structure and Function in CKD. Kidney Int. Rep. 2020, 5, 1052-1060. [CrossRef]

10. You, A.S.; Kalantar-Zadeh, K.; Lerner, L.; Nakata, T.; Lopez, N.; Lou, L.; Veliz, M.; Soohoo, M.; Jing, J.; Zaldivar, F.; et al. Association of Growth Differentiation Factor 15 with Mortality in a Prospective Hemodialysis Cohort. Cardiorenal. Med. 2017, 7, 158-168. [CrossRef] [PubMed]

11. Carney, E.F. The impact of chronic kidney disease on global health. Nat. Rev. Nephrol. 2020, 16, 251. [CrossRef]

12. Shoemaker, M.B.; Stevenson, W.G. The ABC death risk score: Is it time to start measuring GDF-15? Eur. Heart J. 2018, 39, 486-487. [CrossRef] [PubMed]

13. Ramspek, C.L.; Voskamp, P.W.; van Ittersum, F.J.; Krediet, R.T.; Dekker, F.W.; van Diepen, M. Prediction models for the mortality risk in chronic dialysis patients: A systematic review and independent external validation study. Clin. Epidemiol. 2017, 9, 451-464 [CrossRef] [PubMed]

14. Yuan, Q.; Zhang, H.; Deng, T.; Tang, S.; Yuan, X.; Tang, W.; Xie, Y.; Ge, H.; Wang, X.; Zhou, Q.; et al. Role of Artificial Intelligence in Kidney Disease. Int. J. Med. Sci. 2020, 17, 970-984. [CrossRef]

15. Chang, J.F.; Wu, C.C.; Hsieh, C.Y.; Li, Y.Y.; Wang, T.M.; Liou, J.C. A Joint Evaluation of Impaired Cardiac Sympathetic Responses and Malnutrition-Inflammation Cachexia for Mortality Risks in Hemodialysis Patients. Front. Med. 2020, 7, 99. [CrossRef] [PubMed]

16. Lin, Y.-L.; Hou, J.-S.; Lai, Y.-H.; Wang, C.-H.; Kuo, C.-H.; Liou, H.-H.; Hsu, B.-G. Association of SARC-F Questionnaire and Mortality in Prevalent Hemodialysis Patients. Diagnostics 2020, 10, 890. [CrossRef]

17. Hijazi, Z.; Oldgren, J.; Lindbäck, J.; Alexander, J.H.; Connolly, S.J.; Eikelboom, J.W.; Ezekowitz, M.D.; Held, C.; Hylek, E.M.; Lopes, R.D.; et al. A biomarker-based risk score to predict death in patients with atrial fibrillation: The ABC (age, biomarkers, clinical history) death risk score. Eur. Heart J. 2018, 39, 477-485. [CrossRef]

18. Khan, S.Q.; Ng, K.; Dhillon, O.; Kelly, D.; Quinn, P.; Squire, I.B.; Davies, J.E.; Ng, L.L. Growth differentiation factor-15 as a prognostic marker in patients with acute myocardial infarction. Eur. Heart J. 2009, 30, 1057-1065. [CrossRef]

19. Eggers, K.M.; Kempf, T.; Venge, P.; Wallentin, L.; Wollert, K.C.; Lindahl, B. Improving long-term risk prediction in patients with acute chest pain: The Global Registry of Acute Coronary Events (GRACE) risk score is enhanced by selected nonnecrosis biomarkers. Am. Heart J. 2010, 160, 88-94. [CrossRef]

20. Anand, I.S.; Kempf, T.; Rector, T.S.; Tapken, H.; Allhoff, T.; Jantzen, F.; Kuskowski, M.; Cohn, J.N.; Drexler, H.; Wollert, K.C. Serial measurement of growth-differentiation factor-15 in heart failure: Relation to disease severity and prognosis in the Valsartan Heart Failure Trial. Circulation 2010, 122, 1387-1395. [CrossRef]

21. Xie, S.; Lu, L.; Liu, L. Growth differentiation factor-15 and the risk of cardiovascular diseases and all-cause mortality: A meta-analysis of prospective studies. Clin. Cardiol. 2019, 42, 513-523. [CrossRef]

22. Breit, S.N.; Carrero, J.J.; Tsai, V.W.; Yagoutifam, N.; Luo, W.; Kuffner, T.; Bauskin, A.R.; Wu, L.; Jiang, L.; Barany, P.; et al. Macrophage inhibitory cytokine-1 (MIC-1/GDF15) and mortality in end-stage renal disease. Nephrol. Dial. Transplant. 2012, 27, 70-75. [CrossRef]

23. Yilmaz, H.; Çelik, H.T.; Gurel, O.M.; Bilgic, M.A.; Namuslu, M.; Bozkurt, H.; Ayyildiz, A.; Inan, O.; Bavbek, N.; Akcay, A. Increased serum levels of GDF-15 associated with mortality and subclinical atherosclerosis in patients on maintenance hemodialysis. Herz 2015, 40 (Suppl. S3), 305-312. [CrossRef] [PubMed]

24. Ravizza, S.; Huschto, T.; Adamov, A.; Böhm, L.; Büsser, A.; Flöther, F.F.; Hinzmann, R.; König, H.; McAhren, S.M.; Robertson, D.H.; et al. Predicting the early risk of chronic kidney disease in patients with diabetes using real-world data. Nat. Med. 2019, 25, 57-59. [CrossRef] [PubMed]

25. Beam, A.L.; Kohane, I.S. Big Data and Machine Learning in Health Care. JAMA 2018, 319, 1317-1318. [CrossRef] [PubMed]

26. Rank, N.; Pfahringer, B.; Kempfert, J.; Stamm, C.; Kühne, T.; Schoenrath, F.; Falk, V.; Eickhoff, C.; Meyer, A. Deep-learning-based real-time prediction of acute kidney injury outperforms human predictive performance. NPJ Digit. Med. 2020, 3, 139. [CrossRef] 